\title{
DIFFERENTIATION OF INTERVAL FUNCTIONS
}

\author{
GUNNAR SCHRÖDER
}

\begin{abstract}
ABstraCr. This paper is concerned with differentiation of interval functions appearing in interval analysis. Two definitions of a derivative are given; the first one uses an isometric restricted imbedding of the quasilinear space of intervals on the real line $R$, and the other definition is independent of that imbedding. Properties of those two concepts are investigated.
\end{abstract}

Interval analysis was initiated by R. E. Moore [6] and has become an important tool in numerical problems. Further basic contributions are those by N. Apostolatos and U. Kulisch [1], E. Hansen [3], F. Krückeberg [4], K. Nickel [7], and others. In the present paper we shall define and consider differentiation of interval functions; by definition, an interval function is a mapping of $I(R)$ into itself, where $I(R)$ is the set of all compact intervals on the real line $R$.

For these intervals we use the notations $A=\left[a_{1}, a_{2}\right], B=\left[b_{1}, b_{2}\right]$, etc., $a .=[a, a]$, etc., and the familiar addition and multiplication $A+B:=$ $\{a+b \mid a \in A, b \in B\}, A \cdot B:=\{a b \mid a \in A, b \in B\}$. The function $d(A, B)=$ $\max \left\{\left|a_{1}-b_{1}\right|,\left|a_{2}-b_{2}\right|\right\}$ defines a metric on $I(R)$, and $\langle I(R), d\rangle$ is a complete metric space. Also $|A|:=d(A, 0$. $)$ is a norm on $I(R)$, but note that there is no inverse operation of + for the whole $I(R)$, which entails that we cannot get a metric from that norm in the usual fashion. Furthermore, we define scalar multiplication $a \circ B:=a \cdot B$. Then $\langle I(R),+, \circ\rangle$ is a quasilinear space in the sense of $O$. Mayer [5].

By a restricted linear mapping $\omega$ of a quasilinear space $Q_{1}$ into a quasilinear space $Q_{2}$ we mean a mapping $\omega: Q_{1} \rightarrow Q_{2}$ satisfying the following conditions.

(1) For all $X, Y \in Q_{1}$ we have $\omega(X+Y)=\omega(X)+\omega(Y)$.

(2) For all $X \in Q_{1}$ and all $a \geqq 0$ we have $\omega(a X)=a \omega(X)$.

The notions of a restricted isomorphism and a restricted imbedding are defined in a similar fashion, $\mathrm{cf}$. [11].

Received by the editors March 13, 1972.

AMS 1970 subject classifications. Primary 46N05; Secondary 28A15, 58C20.

Key words and phrases. Interval analysis, interval functions, quasilinear space, restricted linear mapping, Fréchet differentiation.

(c) American Mathematical Society 1973 
THEOREM 1. There is an isometric restricted imbedding $\pi$ of $\langle I(R),+, \circ, d\rangle$ into the Banach space $\left\langle R^{2},+, \circ, \tilde{d}\right\rangle$, where $\tilde{d}$ is the metric generated by the norm $\left\|\left(a_{1}, a_{2}\right)\right\|=\max \left\{\left|a_{1}\right|,\left|a_{2}\right|\right\}$, and $\circ$ denotes the usual scalar multiplication in $R^{2}$.

Proof. $\quad I(R),+, \circ, d\rangle$ being given, a theorem by $\mathrm{H}$. Rådström [8] implies that there exists a normed vector space and an isometric restricted imbedding of the given space into that vector space. In the present case, the latter is even a Banach space and the mapping is simply $\pi\left[a_{1}, a_{2}\right]=$ $\left(a_{1}, a_{2}\right)$, which is isometric, because

$$
\begin{aligned}
\tilde{d}\left(\pi\left[a_{1}, a_{2}\right], \pi\left[b_{1}, b_{2}\right]\right) & =\tilde{d}\left(\left(a_{1}, a_{2}\right),\left(b_{1}, b_{2}\right)\right) \\
& =\max \left\{\left|a_{1}-b_{1}\right|,\left|a_{2}-b_{2}\right|\right\}=d\left(\left[a_{1}, a_{2}\right],\left[b_{1}, b_{2}\right]\right) .
\end{aligned}
$$

Theorem 1 implies that with any interval function $F: \mathscr{M} \rightarrow I(R)$, $\mathscr{M} \subset I(R)$, we can associate a mapping $\hat{F}: . \widehat{l} \rightarrow R^{2}, \hat{\mathscr{l}} \subset R^{2}$, defined by $\hat{F}:=\pi F \pi^{-1}$ and $\mathscr{H}:=\pi(\mathscr{M})$. On the other hand, the interval function $F$ can be represented by two interval functionals $f_{1}, f_{2}$ on $\mathscr{M}$ such that $F(X)=\left[f_{1}(X), f_{2}(X)\right]$. Modifying and extending ideas used by $\mathrm{H}$. T. Banks and M. Q. Jacobs [2] in connection with set-valued functions, we may define a differentiation for interval functions as follows.

Definition 1. An interval function $F: \mathscr{M} \rightarrow I(R)$ is said to be $\pi$ Fréchet differentiable at a point $X \in \mathscr{M}$ if $F$ is Fréchet differentiable at $\hat{X}=\pi X$, that is, if there exists a linear mapping $d F(X): R^{2} \rightarrow R^{2}$ and a mapping $r: R^{2} \rightarrow R^{2}$ with the following properties.

(1) $r(0,0)=(0,0), \lim _{i: H, \rightarrow 0}\|r(H)\| /\|H\|=0$.

(2) For any $H$ in a sufficiently small neighborhood of $(0,0)$ we have $\hat{F}(\hat{X}+H)-\hat{F}(\hat{X})=d F(X)(H)+r(H)$.

$d F(X)$ is called the $\pi$-Fréchet differential of $F$ at $X$.

The $\pi$-Fréchet differential $d f(X)$ of an interval functional $f$ can be defined in a similar way.

It can be readily seen that $\pi$-Fréchet differentiability of an interval function $F$ implies continuity of $F$.

EXAMPLE. The interval function $F(X)=[-1,2] \cdot X=[-1,2] \cdot\left[x_{1}, x_{2}\right]$ is $\pi$-Fréchet differentiable precisely at those $X$ for which $x_{2}>-2 x_{1}$ or $-2 x_{1}>x_{2}>-x_{1} / 2$ or $-x_{1} / 2>x_{2}$, and we have

$$
\begin{aligned}
& d F(X)(H)=\left(-h_{2}, 2 h_{2}\right) \quad \text { if } x_{2}>-2 x_{1}, \\
& =\left(2 h_{1}, 2 h_{2}\right) \quad \text { if }-2 x_{1}>x_{2}>-x_{1} / 2 \text {, } \\
& =\left(2 h_{1},-h_{1}\right) \text { if }-x_{1} / 2>x_{2} \text {. }
\end{aligned}
$$

Let $\lambda: I(R) \rightarrow R$ be defined by $\lambda X=\hat{\lambda}\left[x_{1}, x_{2}\right]=x_{2}-x_{1}$. Then the following proposition holds. 
Proposition 2. Let $F: \mathscr{M} \rightarrow I(R)$ and be open in $R^{2}$ and suppose that $F$ is $\pi$-Fréchet differentiable at $X \in \mathscr{M}$. Then $d F(X)(H) \in \hat{I}(R)$ if and only if $\lambda F$ is nondecreasing in the direction of $H=\left(h_{1}, h_{2}\right)$, which means that, for all $t$ in a sufficiently small neighborhood of 0 , the function $\lambda . F\left[x_{1}+t h_{1}, x_{2}+t h_{2}\right]$ is a nondecreasing function of $t$.

The proof follows from Corollary 1.4 in [10] by noting that, for fixed $H$, Fréchet differentiation is differentiation with respect to the real parameter $t$.

If interval functions $F_{1}$ and $F_{2}$ are $\pi$-Fréchet differentiable at $X \in \mathscr{M}$, then the interval function $F_{1} \cdot F_{2}$, which is defined by $\left(F_{1} \cdot F_{2}\right)(X)=$ $F_{1}(X) \cdot F_{2}(X)$, need not be $\pi$-Fréchet differentiable at $X$. This is illustrated by the example $F_{1}\left[x_{1}, x_{2}\right]:=\left[\frac{1}{4}, x_{2}\right], F_{2}\left[x_{1}, x_{2}\right]:=\left[x_{1}, x_{2}\right]$ at $[0.1]$. However, the following theorem holds.

THEOREM 3. Let $F_{i}: \mathscr{M} \rightarrow I(R)$, Ill open in $R^{2}$, be $\pi$-Fréchet differentiable at $X \in \mathscr{M}$, where $i=1,2$, and $F_{1}(X)=\left[f_{1}(X), f_{2}(X)\right], F_{2}(X)=\left[f_{3}(X), f_{1}(X)\right]$. Suppose that there exist pairs $(i, j)$ and $(m, n)$, where $i, m=1$ or 2 and $j, n=3$ or 4 , such that, for all $(k, l), k=1$ or $2, l=3$ or $4,(k, l) \neq(i, j)$, $(k, l) \neq(m, n)$, we have

$$
f_{i}(X) f_{j}(X)<f_{k}(X) f_{l}(X)<f_{m}(X) f_{n}(X) .
$$

Then $F_{1} \cdot F_{2}$ is $\pi$-Fréchet differentiable at $X$, and

$$
\begin{array}{r}
d\left(F_{1} \cdot F_{2}\right)(X)(H)=\left(f_{i}(X) d f_{j}(X)(H)+f_{j}(X) d f_{i}(X)(H),\right. \\
\left.f_{m}(X) d f_{n}(X)(H)+f_{n}(X) d f_{m}(X)(H)\right) .
\end{array}
$$

Proof. Since the functions $F_{1}$ and $F_{2}$ are $\pi$-Fréchet differentiable at $X$, they are continuous at $X$. Consequently, in a sufficiently small neighborhood of $X$, the function $F_{1} \cdot F_{2}$ can be represented in the form

$$
\left(F_{1} \cdot F_{2}\right)(Y)=\left[f_{i}(Y) f_{j}(Y), f_{m}(Y) f_{n}(Y)\right]
$$

Furthermore, we have

$$
d\left(f_{i} f_{j}\right)(X)(H)=f_{i}(X) d f_{j}(X)(H)+f_{j}(X) d f_{i}(X)(H) .
$$

From this, the statement follows.

Clearly, there are other representations of an interval $X=\left[x_{1}, x_{2}\right] \in$ $I(R)$ : for instance $X \doteq 1 . \varphi(X)+[-1,1] \lambda(X) / 2$, where $\varphi(X):=\left(x_{1}+x_{2}\right) / 2$; cf. H. Ratschek [9]. This raises the question whether the use of such a representation and a corresponding imbedding of $I(R)$ into $R^{2}$, say, $X \mapsto(\varphi(X), \lambda(X))$, would give the same concept of differentiability. We shall prove that this holds not only for our particular representation, but also for any restricted homeomorphism, as follows. 
THEOREM 4. Let $\gamma$ be a homeomorphic restricted imbedding of $\langle I(R),+, \circ, d\rangle$ into $\left\langle R^{2},+, \circ, \tilde{d}\right\rangle$. Then $F: \mathscr{M} \rightarrow I(R)$, $\mathscr{M}$ open in $R^{2}$, is $\pi$-Fréchet differentiable at $X \in \mathscr{M}$ if and only if the mapping $F^{*}: \mathscr{M}^{*} \rightarrow R^{2}$, $\mathscr{M}^{*}:=\gamma(\mathscr{M})$, which is defined by $F^{*}:=\gamma F \gamma^{-1}$, is Fréchet differentiable at $X^{*}:=\gamma(X)$. Then

$$
d F(X)=\left\langle\pi \gamma^{-1}\right\rangle d F^{*}\left(X^{*}\right)\left\langle\gamma \pi^{-1}\right\rangle
$$

here, $\left\langle\pi \gamma^{-1}\right\rangle$ and $\left\langle\gamma \pi^{-1}\right\rangle$ are the automorphisms induced in $R^{2}$ by $\pi \gamma^{-1}$ and $\gamma \pi^{-1}$, respectively.

Proof. We have $\pi F \pi^{-1}=\pi \gamma^{-1}\left(\gamma F \gamma^{-1}\right) \gamma \pi^{-1}=\pi \gamma^{-1} F^{*} \gamma \pi^{-1}$. We show that, without loss of generality, we may continue $\pi \gamma^{-1}$ to a linear mapping of the whole plane $R^{2}$ into itself. The mapping $\pi \gamma^{-1}$ is restricted linear and injective on $\gamma(I(R))$. If $X \notin \gamma(I(R))$, then $-X \in \gamma(I(R))$. Noting this, we may set

$$
\begin{aligned}
\left\langle\pi \gamma^{-1}\right\rangle(X) & =\pi \gamma^{-1}(X) & & \text { if } X \in \gamma(I(R)), \\
& =-\pi \gamma^{-1}(-X) & & \text { if } X \notin \gamma(I(R)) .
\end{aligned}
$$

This mapping is linear. In fact, for negative $\alpha$ we have

$$
\begin{array}{ll}
\left\langle\pi \gamma^{-1}\right\rangle(\alpha X)=-\pi \gamma^{-1}(-\alpha X)=\alpha \pi \gamma^{-1}(X)=\alpha\left\langle\pi \gamma^{-1}\right\rangle(X) & \text { if } X \in \gamma(I(R)), \\
\left\langle\pi \gamma^{-1}\right\rangle(\alpha X)=\pi \gamma^{-1}(\alpha X)=-\alpha \pi \gamma^{-1}(-X)=\alpha\left\langle\pi \gamma^{-1}\right\rangle(X) & \text { if } X \notin \gamma(I(R)) .
\end{array}
$$

Since any linear mapping is Fréchet differentiable, the chain rule implies

$$
d F(X)=\left\langle\pi \gamma^{-1}\right\rangle d F^{*}\left(X^{*}\right)\left\langle\gamma \pi^{-1}\right\rangle .
$$

This completes the proof.

Using the representation of an interval function $F$ in terms of interval functionals $f_{1}$ and $f_{2}$, one can prove the following analogue of a familiar condition for an extremum.

Proposition 5. Let $F: M \rightarrow I(R)$, . /l open in $R^{2}$, be $\pi$-Fréchet differentiable at $X \in \mathscr{M}$ and suppose that $F(X) \subset F(Y)$ or $F(Y) \subset F(X)$ for all $Y$ in a neighborhood of $X$. Then $d F(X)=0$.

Our Definition 1 of Fréchet differentiability of interval functions uses a particular imbedding of $I(R)$ into $R^{2}$, but Theorem 4 states that this is not essential. In the last part of the paper we propose another concept of differentiability without the use of an imbedding.

A set $\mathscr{M} \subset I(R)$ is called a convex cone in $I(R)$ if $\mathscr{M}$ is the image of a quasilinear space with respect to a restricted isomorphism.

Definition 2. Let $F: \mathscr{M} \rightarrow I(R)$, where $\mathscr{M}$ is a convex cone in $I(R)$. Then $F$ is said to be $Q$-differentiable at $X \in \mathscr{M}$ if there exist a restricted linear mapping $d_{1} F(X): \mathscr{M} \rightarrow I(R)$ and a mapping $r: \mathscr{M} \rightarrow I(R)$ with the following properties. 
(1) $r(0)=.0 ., \lim _{H \rightarrow 0 .} r(H) /|H|=0$.

(2) There exists an $\varepsilon>0$ such that, for all $H \in \mathscr{M},|H|<\varepsilon$,

$$
F(X+H)=F(X)+d_{1} F(X)(H)+r(H) .
$$

$d_{1} F(X)$ is called the $Q$-differential of $F$ at $X$.

It is interesting to note that Definition 2 is not a generalisation of Definition 1 and conversely. However, under an additional assumption we may establish a relation between the two definitions, as follows.

THEOREM 6. Let $F: \mathscr{M} \rightarrow I(R)$, where $\mathscr{M}$ is an open convex cone in $I(R)$. Suppose that $F$ is $\pi$-Fréchet differentiable at $X \in \mathscr{M}, X \neq a$, and $d F(X)(H) \in$ $\hat{I}(R)$ for $H \in \mathscr{M}$. Then $F$ is $Q$-differentiable at $X$, and

$$
d_{1} F(X)=\pi^{-1} d F(X) \pi \mid \mathscr{M} .
$$

Proof. It can be shown that if $d_{1} F(X)$ exists, it is unique. Furthermore, for $H \in \mathscr{M}$ we have

$$
\hat{F}(\hat{X}+\hat{H})=\hat{F}(\hat{X})+d F(X)(\hat{H})+r(\hat{H}) .
$$

Application of $\pi^{-1}$ gives

$$
F(X+H)=F(X)+\pi^{-1} d F(X) \pi(H)+\pi^{-1} r \pi(H) .
$$

$\pi^{-1} d F(X) \pi: \mathscr{M} \rightarrow I(R)$ is restricted linear, and $\pi^{-1} r \pi(0)=0$.

$$
\lim _{H \rightarrow 0 .} \pi^{-1} r \pi(H) /|H|=0 .
$$

This completes the proof.

The proposed concept of differentiation may serve as a basis of a calculus for interval functions, which is often needed in solving numerical problems with the help of interval analysis.

\section{REFERENCES}

1. N. Apostolatos and U. Kulisch, Grundlagen einer Maschinenintervallarithmetik, Computing (Arch. Elektron. Rechnen) 2 (1967), 89-104. MR 39 \#1077.

2. H. T. Banks and M. Q. Jacobs, A differential calculus for multifunctions, J. Math. Anal. Appl. 29 (1970), 246-272. MR $42 \# 846$.

3. E. Hansen, Interval arithmetic in matrix computations. I, J. Soc. Indust. Appl. Math. Ser. B. Numer. Anal. 2 (1965), 308-320. MR 32 \#4833.

4. F. Krückeberg, Numerische Intervallrechnung und deren Anwendung, Bonn, 1966.

5. O. Mayer, Algebraische und metrische Strukturen in der Intervallrechnung und einige Anwendungen, Computing (Arch. Elektron. Rechnen) 5 (1970), 144-162. MR 43 \#2871.

6. R. E. Moore, Interval analysis, Prentice-Hall, Englewood Cliffs, N.J., 1966. MR 37 \#7069. 
7. K. Nickel, Über die Notwendigkeit einer Fehlerschranken-Arithmetik für Rechenautomaten, Numer. Math. 9 (1966), 69-79. MR 34 \#5334.

8. H. Rådström, An embedding theorem for spaces of convex sets, Proc. Amer. Math. Soc. 3 (1952), 165-169. MR 13, 659.

9. H. Ratschek, Die binären Systeme der Intervallarithmetik, Computing (Arch. Elektron. Rechnen) 6 (1970), 295-308.

10. H. Ratschek and G. Schröder, Über die Ableitung von intervallwertigen Funktionen, Computing (Arch. Elektron. Rechnen) 7 (1971), 172-187.

11. _ U Über den quasilinearen Raum (to appear).

Mathematisches Institut, Universität Karlsruhe, Karlsruhe, Germany 